\title{
A simple monomer-based model-Hamiltonian approach to combine excitonic coupling and Jahn-Teller theory
}

\author{
Pablo García-Fernández, ${ }^{1}$ Ljubica Andjelković, ${ }^{2}$ Matija Zlatar, ${ }^{2}$ Maja Gruden-Pavlović,,${ }^{3, a)}$ \\ and Andreas Dreuw ${ }^{4, a)}$ \\ ${ }^{1}$ Ciencias de la Tierra y Física de la Materia Condensada, Universidad de Cantabria, \\ Santander, Spain \\ ${ }^{2}$ Center for Chemistry, IHTM, University of Belgrade, Studentski trg 12-16, Belgrade, Serbia \\ ${ }^{3}$ Faculty of Chemistry, University of Belgrade, Studentski trg 12-16, Belgrade, Serbia \\ ${ }^{4}$ Interdisciplinary Center for Scientific Computing, Ruprecht-Karls-University, Im Neuenheimer Feld 368, \\ 69120 Heidelberg, Germany
}

(Received 4 September 2013; accepted 16 October 2013; published online 4 November 2013)

\begin{abstract}
The interplay of excitonic and vibronic coupling in coupled chromophores determines the efficiency of exciton localization vs delocalization, or in other words, coherent excitation energy transfer vs exciton hopping. For the investigation of exciton localization in large coupled dimers, a model Hamiltonian approach is derived, the ingredients of which can all be obtained from monomer $a b$ initio calculations alone avoiding costly $a b$ initio computation of the full dimer. The accuracy and applicability of this model are exemplified for the benzene dimer by rigorous comparison to ab initio results. (C) 2013 AIP Publishing LLC. [http://dx.doi.org/10.1063/1.4827398]
\end{abstract}

\section{INTRODUCTION}

Conjugated $\pi$-systems are deservedly the subject of extensive experimental and theoretical studies, considering that $\pi$-conjugated smart materials have become increasingly important in everyday life. ${ }^{1-13} \mathrm{~A}$ comprehensive collection of their electronic spectra and electronic and structural properties has been presented over decades, with regard to their remarkable applicability in organic light-emitting diodes (OLED), ${ }^{14}$ in semi-conducting layers of field-effect transistors (FET), 1,12,15,16 as well as in organic solar cells which are being developed as cheap substitutes for silicon-based solar cells. However, the low energy conversion efficiency of organic solar cells is the main bottleneck, which still limits their usage. This is partially due to rather inefficient excitation energy transport (EET) $)^{11,17}$ within the organic materials. For designing improved organic materials, all occurring processes and the effects determining their efficiency need to be ascertained and eventually well understood.

To investigate EET pathways in organic materials theoretically, it is an important first step to compute the excitonic coupling (EC) between individual chromophores using quantum chemical methods for the excited states of the system, because the qualitative character and the quantitative rate of EET are determined by EC. ${ }^{18-20}$ EET usually occurs from an electronically excited molecule to a non-excited spatially close molecule with lower excitation energy via Förster (Coloumb) or Dexter (exchange)-type or both transfer mechanisms between excitonically coupled dimers. ${ }^{21}$ In general, two types of EET are distinguished, coherent and in-coherent EET. $^{22}$ The first is usually understood in terms of delocalized

\footnotetext{
a) Authors to whom correspondence should be addressed. Electronic addresses: gmaja@chem.bg.ac.rs and dreuw@uni-heidelberg.de
}

excited electronic states over several chromophores and the monomer units remain structurally identical. The latter one is often described as "hopping" of excitation energy from one site to the other, i.e., the exciton is localized on one monomer unit due to its geometry relaxation and then transferred dynamically to a neighboring site. However, most real cases of practical importance belong to intermediate situations.

In order to understand the geometry relaxation of an electronically excited dimer, or equivalently, the localization of the exciton, it is necessary to explicitly take nuclear motion into account. Many theories exist for the description of EET processes, ${ }^{22}$ however, only a very few studies are available that consider both electronic and vibrational contributions to the energy transfer process in a rigorous, quantum mechanical way. ${ }^{20,23-25}$ Typically, the effect of vibrations is often included as dynamic disorder in the electronic coupling. ${ }^{26,27}$

The coupling between electronic and vibrational degrees of freedom is generally quantified by the so-called nonadiabatic couplings, ${ }^{28}$ and structural, symmetry-breaking distortions can be explained in this framework by Jahn-Teller (JT) and pseudo Jahn-Teller (PJT) theory. Hence, the major goal of this work is to understand the localization mechanism of excited states and the driving forces responsible for it, in the framework of the established PJT formalism and Förster's degenerate perturbation theory approach. ${ }^{29} \mathrm{We}$ develop models for the reliable description, interpretation, and prediction of excitonic coupling taking into account vibronic coupling, and the interplay between these two effects. Most importantly, the necessary quantities for the dimer Hamiltonian can be derived from monomer calculations alone. The proposed theory is validated using the benzene dimer as test case, since it presents one of the simplest, aromatic systems with $\pi-\pi$ interaction, representative of more complex chromophores. In addition, extensive experimental and 
theoretical data are available in the bibliography, with which we can compare our computed data. ${ }^{30-39}$ For the purpose of this work, time-dependent density functional theory (TDDFT) was chosen as standard method for the calculation of the excited states, due to its computational efficiency. Its accuracy is here clearly sufficient despite its well-known drawbacks. $^{40}$

A detailed understanding of the physical effects determining the electronic properties of the benzene dimer, its excited states, and the role of vibronic coupling is an essential prerequisite for the successful investigation of more complex systems. As it will be shown, the new developed model predicts the properties of the dimer through the simple interpretation of the monomer characteristics and its intrinsic features, which can eventually be used to study much larger systems of biological and technological interest.

\section{THEORETICAL BACKGROUND-VIBRONIC MODEL FOR EXCITONIC COUPLING}

In order to develop a theory, which takes excitonic and vibronic coupling into account, and to understand their mutual influence at least in a qualitative manner, one needs to start with some basic assumptions: (a) It is assumed that the monomer is a closed-shell species (see Figure 1(a)) which should be correct for most systems not containing transition metal ions. (b) The first excited state of the monomer is mainly described by a single excitation from the highest occupied molecular orbital (HOMO) to the lowest unoccupied one (LUMO). While this condition implies a very small configuration interaction, this later condition is reasonable if excited states with other configurations are sufficiently separated from the HOMO-LUMO ones. (c) In order to take advantage of a JT-type formulation it is assumed that some kind of symmetry element relating the two monomers in a dimer exists. In the case of the benzene dimer shown in Figure 1(b) this operation can be taken to be a reflection or inversion but the theory is not limited to them in its application to other

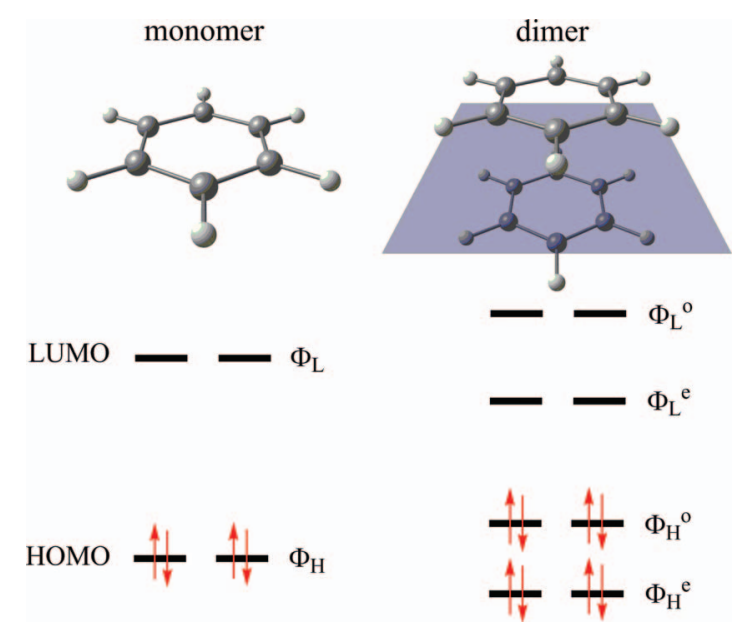

FIG. 1. Scheme of the frontier orbitals of the monomer and the dimer, in which, a symmetry operation relates the monomer orbitals to the dimer ones. Hence it is possible to classify the dimer orbitals accordingly, here with respect to the coplanar reflection plane.

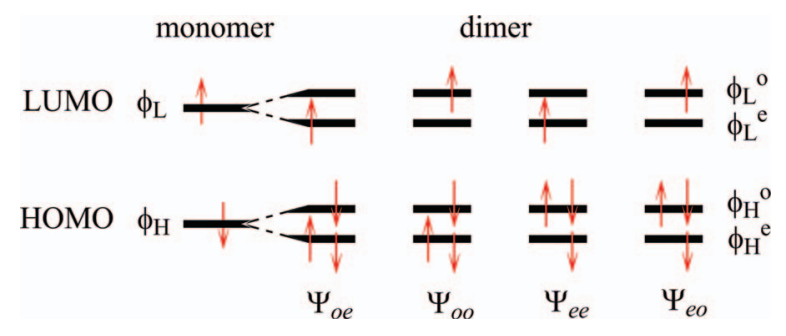

FIG. 2. Scheme of all singly excited determinants within the dimer orbital space composed only of linear combinations of the HOMOs and the LUMOs of the monomer units, and the notation used to differentiate the different excited states arising from it.

cases as, for example, it could be applied to an n-monomer system with a $C_{n}$ axis in the system.

Let us first discuss the nature of the lowest excited states in a symmetric dimer. For simplicity, it is first supposed that monomers HOMO and LUMO are non-degenerate, however this restriction can be easily lifted and the solutions for the degenerate case will be given also below for benzene. Using the symmetry condition (c) we can write the dimer orbitals as symmetrized combinations of the monomer orbitals:

$$
\begin{aligned}
\Phi_{H}^{\alpha} & =\frac{1}{\sqrt{2\left(1 \pm S_{H}\right)}}\left(\Phi_{H}^{1} \pm \Phi_{H}^{2}\right) \\
\Phi_{L}^{\alpha} & =\frac{1}{\sqrt{2\left(1 \pm S_{L}\right)}}\left(\Phi_{L}^{1} \pm \Phi_{L}^{2}\right) .
\end{aligned}
$$

In the equations above the sign corresponds to the character of the wavefunction with respect to the considered symmetry operation. We will call the combination with the + sign even $(\alpha=e)$ and the combination with the - sign odd ( $\alpha=o$ ). It is important to note that the even and odd notations here refer exclusively to the main symmetry operation and they should not be understood as proper even or odd character with respect to inversion in real space which, in many cases, the studied molecules lack. The symbols $S_{H}$ and $\mathrm{S}_{L}$ denote the HOMO-HOMO and LUMO-LUMO orbital overlaps. Note that in deriving expressions (1) and (2) we are neglecting the interactions of the HOMO of a monomer with the LUMO of the other, and vice versa. This is justified, because the HOMO-LUMO interaction is small. Using conditions (a) and (b) we can now generate all singly excited determinants that give rise to the lowest dimer singlet excited states (Figure 2), which can be classified with respect to the position of the electron and hole of the exciton, as well as from a symmetry point-of-view.

In a second step we are going to take the structural distortion into account that occurs on the monomer when the system is excited into the excited state. If we describe this distortion by an effective mode $Q$, using symmetry, we can construct two distortion modes for the dimer:

$$
\begin{aligned}
& Q_{e}=\frac{1}{\sqrt{2}}\left(Q_{1}+Q_{2}\right), \\
& Q_{o}=\frac{1}{\sqrt{2}}\left(Q_{1}-Q_{2}\right) .
\end{aligned}
$$


Here, and similar to previous notation, $Q_{e}$ and $Q_{o}$ have +1 and -1 characters with respect to the symmetry operation, and $Q_{1}$ and $Q_{2}$ are local distortions in each of the monomers. In Figure 3 we give a schematic representation of these modes for the benzene dimer in a $D_{6 h}$ configuration.

Finally we will build the system Hamiltonian as a sum of electronic, vibrational, and vibronic parts. The electronic contribution $\left(H_{e l}(\{\vec{r}\} ; R)\right)$ depends on the electron coordinates, $\{\vec{r}\}$, and the distance between the monomers, $R$, the vibra- tional on the distortion coordinates $\left(Q_{e}, Q_{o}\right)$ while the vibronic part depends on both:

$$
\begin{aligned}
H= & H_{e l}(\{\vec{r}\} ; R)+H_{o}^{\prime}(\{\vec{r}\} ; R) Q_{o}+H_{e}^{\prime}(\{\vec{r}\} ; R) Q_{e} \\
& +H_{v}\left(Q_{e}, Q_{o} ; R\right) .
\end{aligned}
$$

Using this Hamiltonian we can now create a vibronic matrix $\left\langle\Psi_{i}|H| \Psi_{j}\right\rangle$ using the states shown in Figure 2. This matrix can be simplified using symmetry to determine only non-zero elements to yield where we have defined the excitonic coupling constants $\Delta_{i}$ $=\left\langle\Psi_{i}|H| \Psi_{i}\right\rangle$ and the diagonal $\mathrm{F}_{i}=\left\langle\Psi_{i}\left|d H / d Q_{\alpha}\right| \Psi_{i}\right\rangle$ and off-diagonal $F_{i}^{j}=\left\langle\Psi_{i}\left|d H / d Q_{\alpha}\right| \Psi_{j}\right\rangle$ vibronic coupling constants (here the index $i$ and $j$ run along the states $e e, o o, e o$, and $o e$ as defined in Figure 2 and $\alpha$ stands for e or o). Using the singlet wavefunctions of the electronic configurations and taking into account that the diagonal terms represent the force exerted by the excited electrons with respect to the ground state, we can get the following expressions for the diagonal vibronic coupling elements:

$$
F_{e e}=F_{o o}=F_{e o}=F_{o e}=-\left\langle\Phi_{H}^{e}\left|\hat{f}_{e}\right| \Phi_{H}^{e}\right\rangle+\left\langle\Phi_{L}^{e}\left|\hat{f}_{e}\right| \Phi_{L}^{e}\right\rangle,
$$

in which $\hat{f}_{e}$ represents the vibronic operator for a singleelectron along $Q_{e}$. Thus, we observe that all diagonal vibronic coupling elements are approximately the same. We can operate in a similar way to find expressions for the off-diagonal elements:

$$
\begin{gathered}
F_{e e}^{o o}=F_{e o}^{o e}=0 \\
F_{e e}^{e o}=F_{o o}^{o e}=\left\langle\Phi_{L}^{e}\left|\hat{f}_{o}\right| \Phi_{L}^{o}\right\rangle,
\end{gathered}
$$
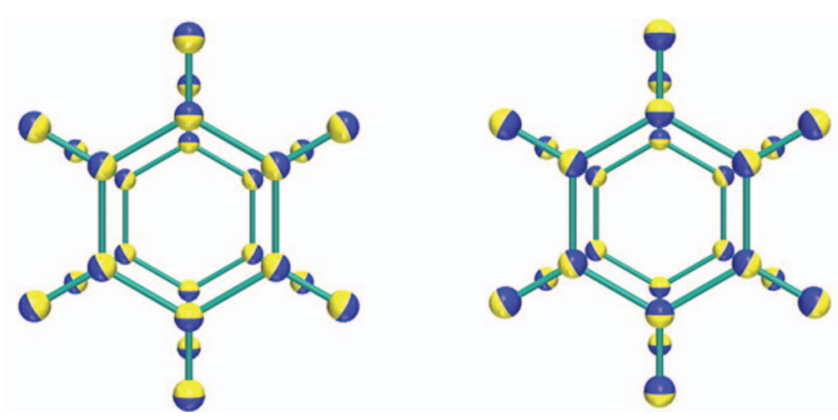

FIG. 3. Schematic representation of symmetric $Q_{e}$ (left) and anti-symmetric distortion $Q_{o}$ (right) of the benzene dimer. The size of vibrational motion on a nucleus is represented as sphere with the radius depending on the magnitude of the motion. The different colours indicate the direction of the motion.

$\left.\begin{array}{ccc}F_{e e}^{o o} Q_{e} & F_{e e}^{e o} Q_{o} & F_{e e}^{o e} Q_{o} \\ \Delta_{o o}+F_{o o} Q_{e} & F_{o o}^{e o} Q_{o} & F_{o o}^{o e} Q_{o} \\ F_{o o}^{e o} Q_{o} & \Delta_{e o}+F_{e o} Q_{e} & F_{e o}^{o e} Q_{e} \\ F_{o o}^{o e} Q_{o} & F_{e o}^{o e} Q_{e} & \Delta_{o e}+F_{o e} Q_{e}\end{array}\right)$,

$$
F_{e e}^{o e}=F_{o o}^{e o}=\left\langle\Phi_{H}^{e}\left|\hat{f}_{o}\right| \Phi_{H}^{o}\right\rangle .
$$

Expression (7) suggests that the force with respect to the ground state on the dimer comes from the removal of an electron from the HOMO (due to the negative sign) that is placed into the LUMO, while expressions (9) and (10) suggest that they are related to the forces of individual electrons in the LUMO and the HOMO, respectively. Indeed, using expressions (1) and (2) we get integrals like

$$
\begin{aligned}
& \left\langle\Phi_{H}^{e}\left|\hat{f}_{o}\right| \Phi_{H}^{o}\right\rangle \\
& =\frac{1}{\sqrt{2\left(1-S_{H}^{2}\right)}}\left[\left\langle\Phi_{1 H}\left|\hat{f}_{1}\right| \Phi_{1 H}\right\rangle-\left\langle\Phi_{1 H}\left|\hat{f}_{2}\right| \Phi_{1 H}\right\rangle\right] .
\end{aligned}
$$

These integrals can be further simplified taking into account that vibronic operators decay very quickly (typically $\alpha \mathrm{r}^{-3}$ ) to neglect the second term in the sum when compared to the first one. Moreover, the first product, whose meaning is that of a reduction factor due to the delocalization of the electron in between the two individual monomers orbitals, can in fact be simplified since our first-principles calculations indicate that $S_{H}^{2} \ll 1$. Under these conditions we write Eqs. (7) and (9), and (10) in terms of integrals depending just on a single monomer:

$$
\begin{aligned}
F_{e e}=F_{o o}=F_{e o}=F_{o o} & =\frac{1}{\sqrt{2}}\left(-\left\langle\Phi_{H}|\hat{f}| \Phi_{H}\right\rangle+\left\langle\Phi_{L}|\hat{f}| \Phi_{L}\right\rangle\right) \\
& =\frac{1}{\sqrt{2}} f_{m o n o}, \\
F_{e e}^{e o} & =F_{o o}^{o e}=\frac{1}{\sqrt{2}} f_{L}, \\
F_{e e}^{o e} & =F_{o o}^{e o}=\frac{1}{\sqrt{2}} f_{H} .
\end{aligned}
$$


Here $f_{\text {mono }}=f_{L}-f_{H}$ is the force experienced in the monomer by removing an electron from the HOMO and placing it in the LUMO, i.e., the force in the excited state of the monomer, while $f_{L}$ and $f_{H}$ are the individual forces created by adding an electron to the LUMO or removing an electron from the HOMO of a monomer, respectively. These two last quantities can be associated with the force on the monomer along the effective mode $Q$ when an electron is added or removed, i.e., the forces for the anion and the cation. In order to check expressions (12)-(14) and the assumptions leading to them we compared the $a b$ initio forces of the anion and cation (Eqs. (13) and (14)) with that of the neutral excited monomer (given by Eq. (12)) finding a reasonable agreement (see Sec. IV). Acting in a similar way for the vibrational part, and imposing that at long range the frequencies of vibration of the dimer need to be those of the monomer we obtain

$$
K_{e}=K_{o}=K_{\text {mono }} .
$$

Thus, we can finally reduce the full vibronic matrix (6) to

$$
H=\frac{1}{2} K_{\text {mono }}\left(Q_{e}^{2}+Q_{o}^{2}\right)+\left(\begin{array}{cccc}
\Delta_{e e}+\frac{1}{\sqrt{2}} f_{\text {mono }} Q_{e} & 0 & \frac{1}{\sqrt{2}} f_{L} Q_{o} & \frac{1}{\sqrt{2}} f_{H} Q_{o} \\
0 & \Delta_{o o}+\frac{1}{\sqrt{2}} f_{\text {mono }} Q_{e} & \frac{1}{\sqrt{2}} f_{H} Q_{o} & \frac{1}{\sqrt{2}} f_{L} Q_{o} \\
\frac{1}{\sqrt{2}} f_{L} Q_{o} & \frac{1}{\sqrt{2}} f_{H} Q_{o} & \Delta_{e o}+\frac{1}{\sqrt{2}} f_{\text {mono }} Q_{e} & 0 \\
\frac{1}{\sqrt{2}} f_{H} Q_{o} & \frac{1}{\sqrt{2}} f_{L} Q_{o} & 0 & \Delta_{o e}+\frac{1}{\sqrt{2}} f_{\text {mono }} Q_{e}
\end{array}\right) .
$$

Here we see that all vibronic interactions in the dimer can be reduced to quantities obtainable from the monomer. This is a very desirable property since it may allow estimation of the role of vibronic coupling in exciton transport without the need to carry out costly dimer calculations, but to do so is generally advised to validate the model with ab initio calculations. We perform this task in Sec. IV.

We can now analyze the physical content of the derived model. The coupling to $Q_{e}$ occurs on the diagonal of the Hamiltonian and connected with $f_{\text {mono }}$, while, on the other hand, the coupling to $Q_{o}$ is always accompanied by vibronic constants $f_{H}$ and $f_{L}$. Taking into account that $f_{\text {mono }}=f_{L}-f_{H}$, there will be systems in which the coupling to $Q_{e}$ dominates ( $f_{L}$ and $f_{H}$ are very different), while in others the coupling to $Q_{o}$ dominates $\left(f_{L} \sim f_{H} \rightarrow\left|f_{\text {mono }}\right| \ll\left|f_{L}\right|,\left|f_{H}\right|\right)$. The emerging picture is that in the first case the distortion is the same and the solution that dominates the vibronic problem, Eq. (16), is an exciton where electron and hole travel together. On the other hand, the case where $f_{L} \sim f_{H}$ leads to different distortions in the two monomers favors the localization of the electron and hole separately, i.e., formation of polarons. Thus, even though our model does not contain several important key ingredients like electrostatic attraction between hole and electron, it provides a very sensible picture of which conditions would favor the separation of the exciton in polarons. These limit solutions can be obtained from Eq. (16) when the excitonic coupling energy $\Delta$ is much smaller than the vibronic coupling energy $(f \cdot Q)$. In that case the solutions take the form

$$
\Psi_{ \pm}^{e}=\frac{1}{\sqrt{2}}\left(\Psi_{o o} \pm \Psi_{e e}\right)
$$

$$
\Psi_{ \pm}^{o}=\frac{1}{\sqrt{2}}\left(\Psi_{o e} \pm \Psi_{e o}\right)
$$

While the $(+)$ combinations correspond to two excitonically coupled local excited states, the $(-)$ combinations represent two symmetrized charge-transfer (CT) excitations. ${ }^{41}$ Later it will be shown that the CT states do not play a role in the benzene dimer due to their symmetry.

We will now focus on the changes between the above model and the one that arises when the HOMO and LUMO are doubly degenerate, as in the case of benzene. The main difference between these models is that in the latter case many more states are involved (see Figure 4 and compare with Figure 2). Observing the monomer states in Figure 4 we see that, in principle, the vibronic coupling problem is more complicated since vibrations belonging to $a_{1 g}, a_{2 g}$, and $e_{1 g}$ can be involved. However, ab initio calculations indicate that in the benzene molecule the effective mode $Q$ belongs to $a_{l g}$ and as a consequence we will not take into account the other possibilities. When constructing all excited states in the benzene dimer involving only the

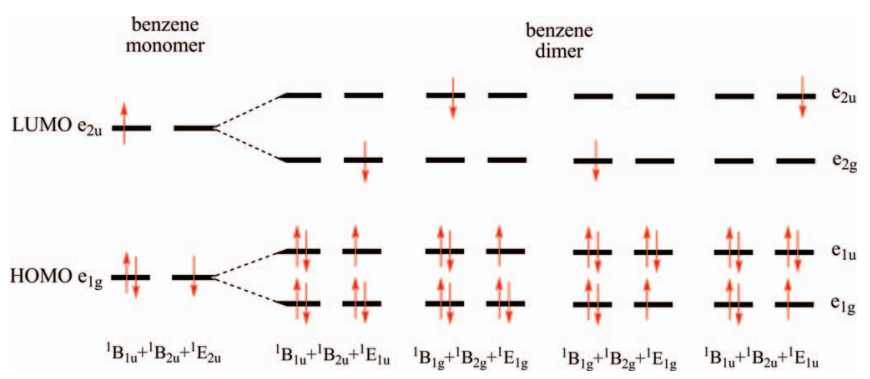

FIG. 4. Singly excited determinants arising from single HOMO-LUMO excitations in the benzene monomer (left) and benzene dimer (right). 
doubly degenerate HOMO and LUMO, we observe that instead of the previous four possibilities now 16 different states are possible. However, since the effective mode in the monomer is $a_{l g}$, the resulting even $\left(Q_{e}\right)$ and odd $\left(Q_{o}\right)$ dimer modes have $a_{1 g}$ and $a_{2 u}$ symmetry, respectively. As a consequence, the lower ${ }^{l} B$ states do not couple with the higher lying ${ }^{l} E$ ones. Therefore, the $E$ states can be com-

$$
\begin{aligned}
H= & \frac{1}{2} K_{\text {mono }}\left(Q_{e}^{2}+Q_{o}^{2}\right) \\
& +\left(\begin{array}{cccc}
\Delta_{1 e}^{e e}+\frac{f_{m} Q_{e}}{\sqrt{2}} & 0 & 0 & 0 \\
0 & \Delta_{1 o}^{o o}+\frac{f_{m} Q_{e}}{\sqrt{2}} & 0 & 0 \\
0 & 0 & \Delta_{1 o}^{e o}+\frac{f_{m} Q_{e}}{\sqrt{2}} & 0 \\
0 & 0 & 0 & \Delta_{1 o}^{o e}+\frac{f_{m} Q_{e}}{\sqrt{2}} \\
0 & 0 & \frac{f_{H}}{\sqrt{2}} Q_{o} & 0 \\
0 & 0 & 0 & \frac{f_{H}}{\sqrt{2}} Q_{o} \\
\frac{f_{L}}{\sqrt{2}} Q_{o} & 0 & 0 & 0 \\
0 & \frac{f_{L}}{\sqrt{2}} Q_{o} & 0 & 0
\end{array}\right.
\end{aligned}
$$$$
\left.\begin{array}{cccc}
0 & 0 & \frac{f_{L}}{\sqrt{2}} Q_{o} & 0 \\
0 & 0 & 0 & \frac{f_{L}}{\sqrt{2}} Q_{o} \\
\frac{f_{H}}{\sqrt{2}} Q_{o} & 0 & 0 & 0 \\
0 & \frac{f_{H}}{\sqrt{2}} Q_{o} & 0 & 0 \\
\Delta_{2 e}^{e e}+\frac{f_{m} Q_{e}}{\sqrt{2}} & 0 & 0 & 0 \\
0 & \Delta_{2 e}^{o o}+\frac{f_{m} Q_{e}}{\sqrt{2}} & 0 & 0 \\
0 & 0 & \Delta_{2 o}^{e o}+\frac{f_{m} Q_{e}}{\sqrt{2}} & 0 \\
0 & 0 & 0 & \Delta_{2 o}^{o e}+\frac{f_{m} Q_{e}}{\sqrt{2}}
\end{array}\right) .
$$
states belong to these $E$ states, they do not play any role in the derived excitonic coupling model due to the symmetry restrictions of the distortion modes. Thus, the dimensionality of the vibronic matrix can be reduced to eight, and using the same approximations as in Eqs. (7)-(14), we find
Note again that, as in the previous case, only information from monomer calculations is required to arrive at a complete description of the benzene dimer coupling Hamiltonian. We will proceed in Sec. IV to verify this model.

\section{COMPUTATIONAL METHODOLOGY}

The underlying computational procedure for the construction of the dimer model Hamiltonian is the following steps:

1. Geometry optimization of the monomer and calculation of vertical excitation energies using different TDDFT levels of theory and comparison with experimental data.

2. Geometry optimization of the monomer in the first excited state and identification of the distortion mode.

3. Choice of mutual orientations of monomer units in the dimer, followed by calculations of vertical excitation energies at different intermolecular separations between two monomer units; selection of particular intermolecular distances from the potential energy curves.

4. Performing calculations along the symmetric $\left(Q_{e}\right)$ and anti-symmetric $\left(Q_{o}\right)$ distortion of the excimer at chosen intermolecular separations.

5. Determination of all parameters in the model Hamiltonian (Eq. (19)); comparison of the results obtained using the model Hamiltonian with ab initio computed ones; validation of proposed model.

As already mentioned, the parameters $f_{H}$ and $f_{L}$ correspond to the forces acting on the monomer HOMO and LUMO at the ground state geometry, and their difference should be practically equal to the force acting on the excited neutral molecule, $f_{\text {mono }}$. These parameters have been obtained pletely neglected in the model. Because the charge transfer

by fitting the curves of HOMO and LUMO energies along the monomer distortion coordinate. $K_{e}$ and $K_{o}$ correspond to the monomer force constant in the excited state and can be extracted by fitting the curves of the first excited state along the particular distortion. $Q_{e}$ and $Q_{o}$ have been constructed over the distortion mode of monomer, $Q_{1}$ and $Q_{2}$.

The structures of the benzene monomer and its dimer were optimized at the level of DFT using the Amsterdam Density Functional program package, ADF2010.01. ${ }^{42-44}$ The hybrid B3LYP functional, ${ }^{45,46}$ with included dispersion, ${ }^{47}$ B3LYP-D3, was used for the symmetry-constrained geometry optimizations. An all electron triple-zeta Slater-type orbitals (STO) plus one polarization function (TZP) basis set was used for all atoms. The difference in energy between two optimized monomers and the dimer, where two monomer units are placed at large intermolecular separation, i.e., there is practically no interaction between them, was $0.0047 \mathrm{eV}$, confirming the size-consistency of the calculations.

Vertical excitation energies of benzene were calculated using TDDFT, ${ }^{40,48,49}$ at B3LYP-D3 optimized geometry with

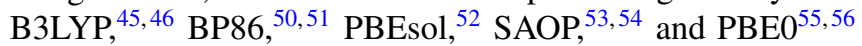
levels of theory. In order to elucidate the best combination of functional and basis set the vertical excitation energies were calculated with DZP, TZP, TZ2P, and QZ4P basis set for all used functionals. Vertical excitation energies and potential energy curves of the excited states of benzene dimer were approximated by adding the excitation energies to the B3LYPD3 ground state.

\section{RESULTS AND DISCUSSION}

In order to study the localization mechanism of the excited benzene dimer and to calculate the model Hamiltonian, 
it is first necessary to compute properties of the benzene monomer to extract the required constants. For this purpose, equilibrium distances and vertical excitation energies have been calculated at different levels of theory and compared with available data. Afterwards, we will present TDDFT results and compare them with the proposed model for the excitonically coupled benzene dimer.

\section{A. The benzene monomer}

The optimized structure of the benzene molecule, in $D_{6 h}$ symmetry, at B3LYP-D3/TZP level of theory, has equilibrium bond lengths values of $r_{\mathrm{CC}}=1.39 \AA$ and $r_{\mathrm{CH}}=1.08 \AA$, in perfect agreement with results of high-level quantum chemical calculations at the coupled cluster single, double (triple) excitations level with correlation-consistent polarized valence quadruple-zeta basis set (CCSD(T)/ccpVQZ). ${ }^{57}$

The excited states of benzene have been the subject of various theoretical and experimental studies. ${ }^{36,58-68}$ The obtained TDDFT values of the excitation energies of the seven lowest excited states of benzene are given in Table I and compared with experimental data. The three well-known absorption bands in the UV spectrum of benzene at 4.90, 6.20, and $6.94 \mathrm{eV}$ are assigned to the transitions ${ }^{1} A_{l g} \rightarrow{ }^{1} B_{2 u},{ }^{1} A_{l g}$ $\rightarrow{ }^{l} B_{1 u}$, and ${ }^{l} A_{l g} \rightarrow{ }^{l} E_{l u}$, respectively. Although, the transitions to $1^{l} B_{2 u}$, and $1^{l} B_{l u}$ excited states, are symmetry for-

TABLE I. Vertical excitation energies of $\mathrm{C}_{6} \mathrm{H}_{6}(\mathrm{eV})$ calculated at the level of TDDFT using different functionals and basis sets. All calculations were performed in $D_{6 h}$ symmetry with $r_{\mathrm{CC}}=1.39 \AA$ and $r_{\mathrm{CH}}=1.08 \AA$.

\begin{tabular}{|c|c|c|c|c|c|c|c|}
\hline \multirow[b]{2}{*}{$\mathrm{E}_{\mathrm{ex}}(\mathrm{eV})$} & \multicolumn{3}{|c|}{ Singlet $\pi \rightarrow \pi^{*}$} & \multicolumn{4}{|c|}{ Triplet $\pi \rightarrow \pi^{*}$} \\
\hline & $1^{1} B_{1 u}$ & $1^{1} B_{2 u}$ & $1^{l} E_{l u}$ & $1^{3} B_{1 u}$ & $1^{3} E_{1 u}$ & $1^{3} B_{2 u}$ & $1^{3} E_{2 g}$ \\
\hline \multicolumn{8}{|l|}{ B3LYP } \\
\hline DZP & 5.53 & 6.34 & 7.31 & 4.07 & 4.84 & 5.23 & 7.23 \\
\hline $\mathrm{TZP}$ & 5.41 & 6.18 & 7.14 & 4.01 & 4.76 & 5.13 & 6.56 \\
\hline TZ2P & 5.43 & 6.18 & 7.14 & 4.02 & 4.77 & 5.14 & 6.57 \\
\hline QZ4P & 5.44 & 6.15 & 6.99 & 4.04 & 4.76 & 5.11 & 6.24 \\
\hline \multicolumn{8}{|l|}{ BP86 } \\
\hline DZP & 5.34 & 6.21 & 7.20 & 4.46 & 4.83 & 5.10 & 7.49 \\
\hline $\mathrm{TZP}$ & 5.26 & 6.08 & 7.05 & 4.41 & 4.77 & 5.02 & 6.77 \\
\hline TZ2P & 5.27 & 6.08 & 7.05 & 4.42 & 4.77 & 5.03 & 6.77 \\
\hline QZ4P & 5.29 & 6.07 & 6.98 & 4.43 & 4.77 & 5.02 & 6.47 \\
\hline \multicolumn{8}{|l|}{ PBEsol } \\
\hline DZP & 5.34 & 6.22 & 7.20 & 4.47 & 4.83 & 5.11 & 7.44 \\
\hline TZP & 5.27 & 6.09 & 7.06 & 4.42 & 4.77 & 5.03 & 6.70 \\
\hline TZ2P & 5.27 & 6.09 & 7.05 & 4.43 & 4.78 & 5.04 & 6.71 \\
\hline QZ4P & 5.29 & 6.06 & 6.96 & 4.43 & 4.78 & 5.02 & 6.32 \\
\hline \multicolumn{8}{|l|}{ SAOP } \\
\hline DZP & 5.32 & 6.20 & 7.16 & 4.44 & 4.81 & 5.09 & 7.14 \\
\hline TZP & 5.28 & 6.09 & 7.05 & 4.42 & 4.78 & 5.04 & 6.59 \\
\hline TZ2P & 5.28 & 6.10 & 7.05 & 4.43 & 4.79 & 5.05 & 6.61 \\
\hline QZ4P & 5.30 & 6.07 & 6.96 & 4.43 & 4.78 & 5.03 & 6.56 \\
\hline \multicolumn{8}{|l|}{ PBE0 } \\
\hline DZP & 5.58 & 6.36 & 7.35 & 3.98 & 4.86 & 5.27 & 7.52 \\
\hline TZP & 5.51 & 6.25 & 7.22 & 3.94 & 4.81 & 5.20 & 6.87 \\
\hline $\mathrm{TZ2P}$ & 5.51 & 6.24 & 7.21 & 3.95 & 4.81 & 5.20 & 6.89 \\
\hline QZ4P & 5.53 & 6.22 & 7.14 & 3.97 & 4.81 & 5.17 & 6.54 \\
\hline Experiment $^{58-62}$ & 4.90 & 6.20 & 6.94 & 3.94 & 4.76 & 5.60 & $6.83 / 7.24$ \\
\hline
\end{tabular}

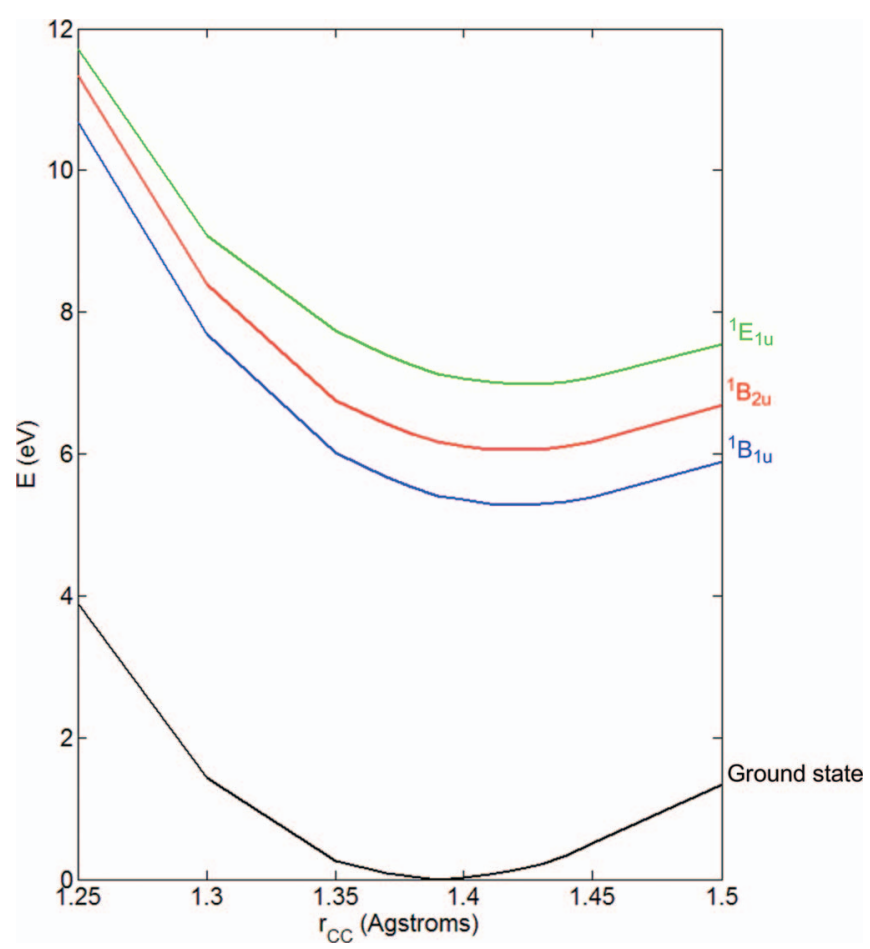

FIG. 5. Potential energy curves of the ground and the three lowest excited singlet states of benzene along its totally symmetric vibrational breathing mode.

bidden in benzene, ${ }^{69}$ due to vibronic interactions they still occur in the experimental spectrum. ${ }^{67}$ The excitation energies are calculated at different levels of theory. The results are consistent to each other, and to previous theoretical considerations, ${ }^{68}$ and do not show large basis set or exchange/correlation functional dependence (Table I). For further studies we have chosen TD-B3LYP/TZP level, although it is clear that it does not exhibit the necessary accuracy to perform a quantitative state-of-the-art study of the excited states of benzene. ${ }^{68}$ Qualitatively same results could be obtained with other levels of theory. However, the focus of the present study is not the investigation of the excited states of benzene, but the rationalization of the electronic structure of the excited benzene dimer and the verification of the proposed model Hamiltonian approach. It is also important to note that the CT problem of TDDFT is irrelevant here, since the CT states are excluded from the investigation based on their symmetry and the fact that they do thus not play a role in the excitonic coupling.

TDDFT geometry optimization, without constraining the symmetry, has shown that the equilibrium structure of the first excited singlet state has $D_{6 h}$ symmetry and bond lengths of $r_{\mathrm{CC}}=1.43 \AA$ and $r_{\mathrm{CH}}=1.08 \AA$. It is obvious that the structural relaxation, which leads from the ground state geometry of benzene to the equilibrium geometry of the first excited state, occurs along the totally symmetric breathing mode. Following this breathing mode from the ground state geometry, the potential energy curves of the three lowest singlet excited electronic states of benzene, in $D_{6 h}$ symmetry, have been computed (Figure 5). These three states of ${ }^{l} B_{1 u},{ }^{l} B_{2 u}$, and ${ }^{l} E_{1 u}$ symmetry are the lowest singlet states arising from HOMOLUMO excitations. 


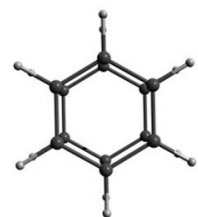

$D_{6 h}$

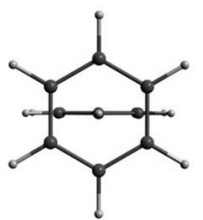

$C_{2 v}$

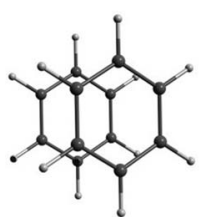

$C_{2 h}$

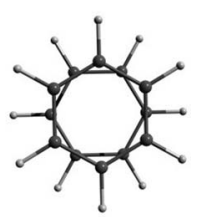

$D_{6} / D_{6 d}$
FIG. 6. Investigated mutual orientations of the benzene dimer.

In order to verify the applicability and accuracy of the proposed model Hamiltonian, which requires only intrinsic features of the constituting monomer to reproduce the excited states of the dimer, we have calculated the necessary parameters $f_{H}$ and $f_{L}$ and obtained values of $14.53 \mathrm{eV} / \AA$ and $23.30 \mathrm{eV} / \AA$, respectively. According to the model, the difference between these forces $(8.77 \mathrm{eV} / \AA)$ acting on the monomer cation on removal of an electron from the HOMO or on the anion on addition of an electron to the LUMO, perfectly corresponds to the force $(8.48 \mathrm{eV} / \AA)$ acting on the excited neutral monomer.
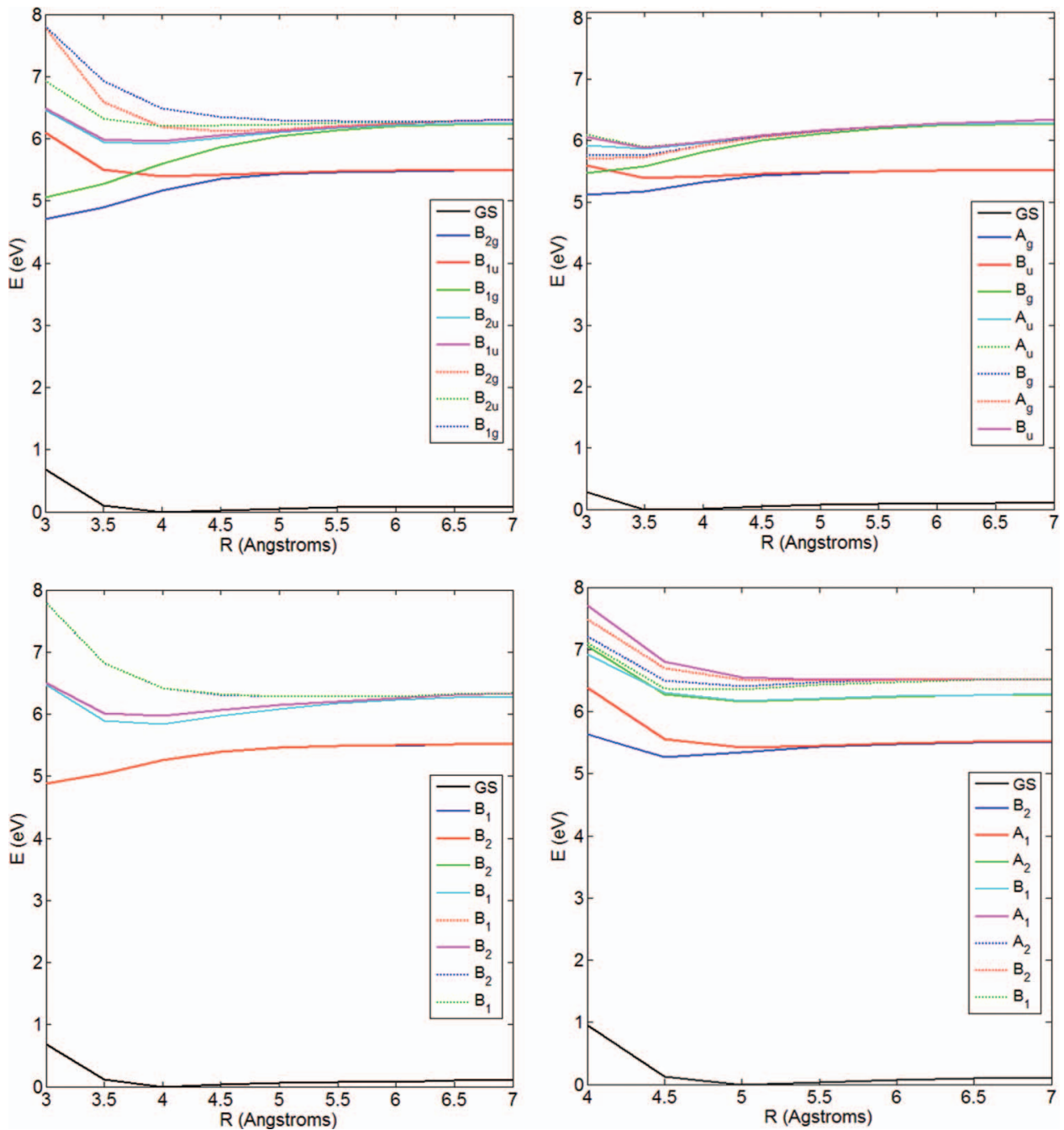

FIG. 7. Potential energy curves along the intermolecular separation coordinate $R$ of the benzene dimer in $D_{6 h}$ (top left), $C_{2 h}$ (top right), $D_{6}$ (bottom left), and $C_{2 v}$ (bottom right) orientation with $r_{\mathrm{CC} 1}=r_{\mathrm{CC} 2}=1.39 \AA$ at the theoretical level of TDDFT/B3LYP-D3. 

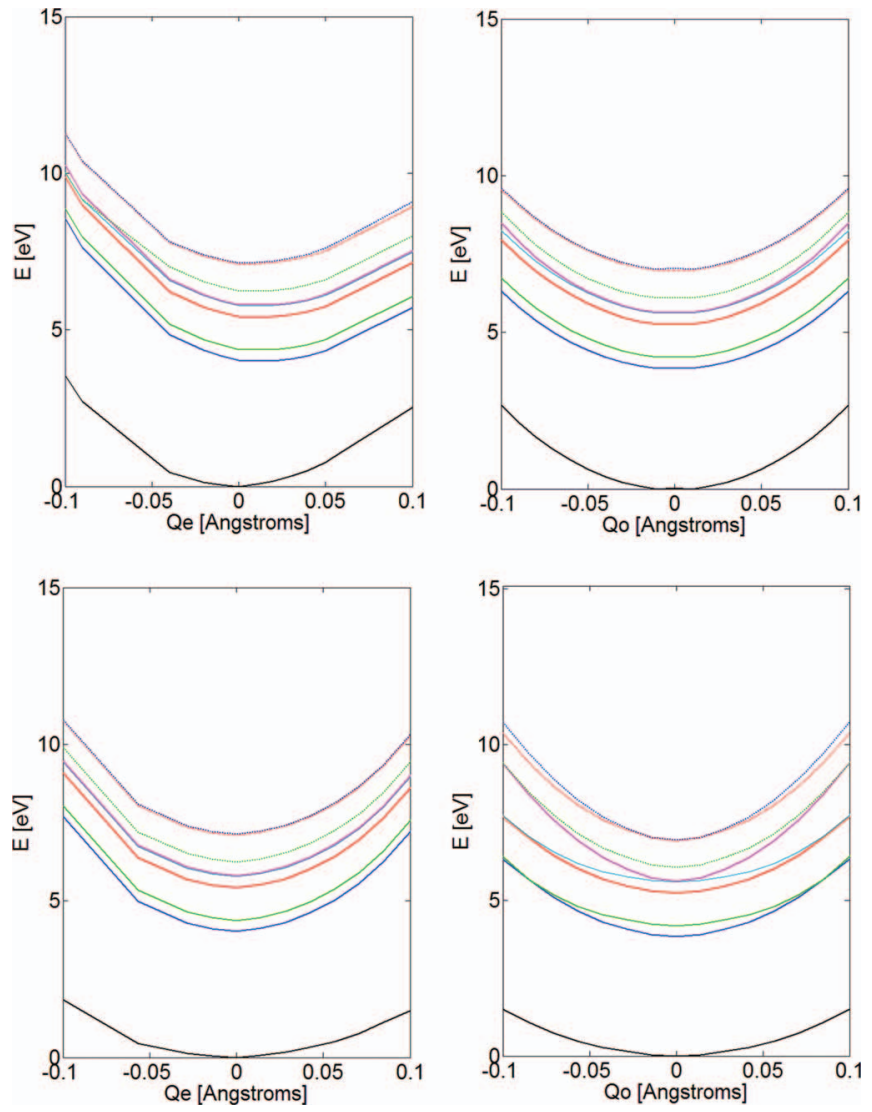

FIG. 8. Potential energy curves of the lowest excited states along the symmetric and the anti-symmetric distortion coordinates $Q_{e}$ (left) and $Q_{o}$ (right) at an intermolecular separation $R$ of $3 \AA$ of the $D_{6 h}$ benzene dimer; TDDFT/B3LYP-D3 calculated plots are given on the top, while the plots obtained using the model Hamiltonian are given at the bottom.

presence of another benzene molecule at this intermolecular distance. The optimal intermolecular separation between two benzene units, $R_{e q}$, is $3.89 \AA$ for $D_{6 h}, 3.63 \AA$ for $C_{2 h}$, $4.97 \AA$ for $C_{2 v}$, and $3.82 \AA$ for $D_{6}$ at the theoretical level of DFT/B3LYP-D3, with splittings of the first two excited states of $0.28 \mathrm{eV}$ for $D_{6 h}, 0.04 \mathrm{eV}$ for $C_{2 h}, 0.08 \mathrm{eV}$ for $C_{2 v}$, and $0.00 \mathrm{eV}$ for $D_{6}$ minima structures, respectively, at the theoretical level of TDDFT/B3LYP (Figure 7).

The potential energy curves of the energetically lowest electronic states of all studied dimer orientations along the intermolecular separation coordinate $R$, going from $7 \AA$ to $3 \AA$ in steps of $0.5 \AA$, are shown in Figure 7. At distances $R<3 \AA$ strong repulsive Coulomb interaction appears resulting in a steep increase of the energies of the electronic states and consequently in their mixing.

The potential energy curves of the electronic states of the parallel, eclipsed, $D_{6 h}$, benzene dimer at various intermolecular separations, $R$ (Figure 7, top left), show that the two first excited states are practically degenerate at intermolecular distances larger than $5 \AA$. At separations $<5 \AA$, a significant excitonic splitting of these states becomes apparent. With decreasing monomer distances, the splitting becomes more significant, and at $R=3 \AA$, it has a value of $1.39 \mathrm{eV}$. In the other studied dimer orientations, the splitting of the first two excited states at $R=3 \AA$ is smaller. In the parallel-displaced orientation, $C_{2 h}$, it has a value of $0.47 \mathrm{eV}$ (Figure 7, top right),
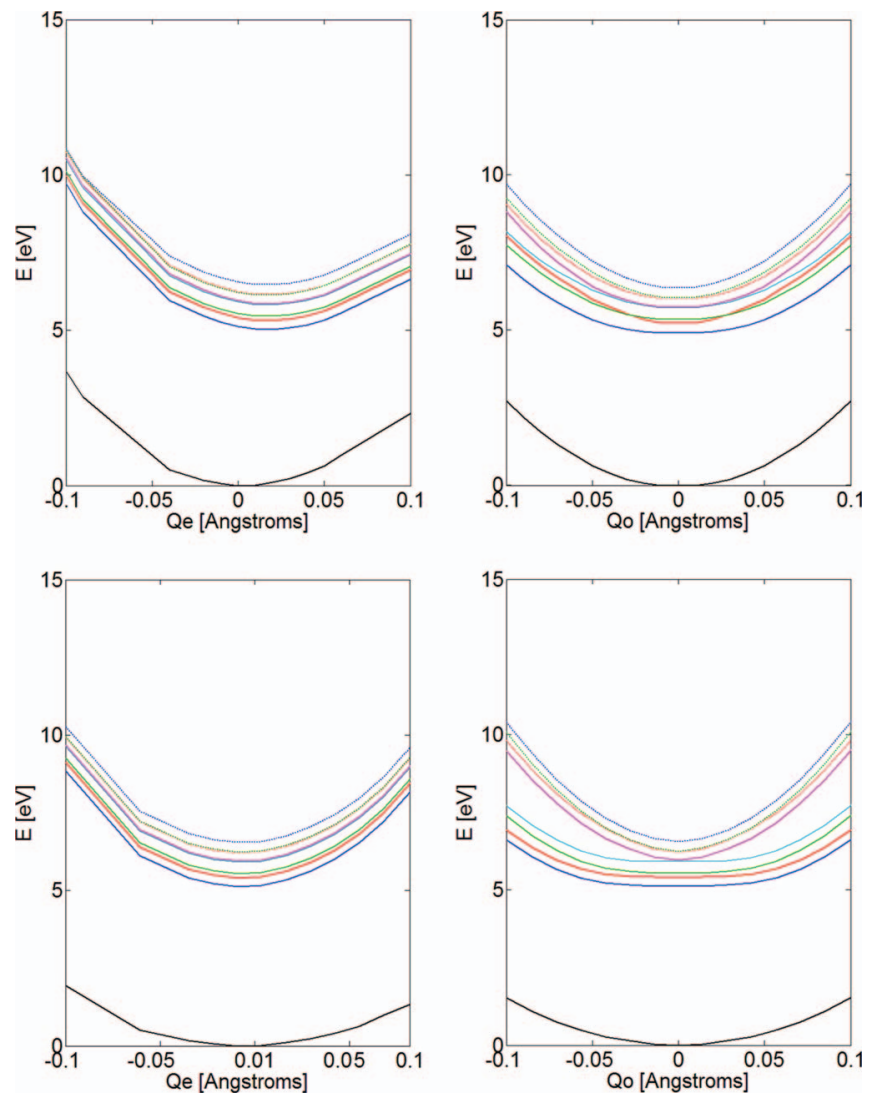

FIG. 9. Potential energy curves of the lowest excited states along the symmetric and the anti-symmetric distortion coordinates $Q_{e}$ (left) and $Q_{o}$ (right) at an intermolecular separation $R$ of $3.89 \AA$ of the $D_{6 h}$ benzene dimer; TDDFT/B3LYP-D3 calculated plots are given on the top, while the plots obtained using the model Hamiltonian are given at the bottom.

while in the $D_{6}$ orientation, the splitting is completely absent (Figure 7, bottom left). In the T-shaped orientation (Figure 7 , bottom right), the splitting of first two excited states is $0.07 \mathrm{eV}$ at an intermolecular separations of $4.5 \AA$.

The largest EC, i.e., the most pronounced splitting of the excited states, appears in the $D_{6 h}$ parallel sandwich orientation. It has been shown previously that a minimum on the potential energy surface of the lowest excited state exists in this $D_{6 h}$ orientation corresponding to a benzene excimer $\left({ }^{l} B_{2 g}\right)$ with an intermolecular separation of only $3.05 \AA .{ }^{72}$ Hence, we studied the localization of the exciton in this parallel $D_{6 h}$ conformation. The relevant potential energy curves of the benzene dimer were determined at fixed values of $R(3 \AA$-excimer minimum, $3.89 \AA$-ground state minimum, and $5 \AA$-borderline case) by following the symmetric and anti-symmetric combinations of the breathing mode, which belong to $a_{1 g}$ and $a_{2 u}$ irreducible representations in the $D_{6 h}$ point group, by varying the bond distance $r_{\mathrm{CC}}$ from 1.25 to $1.50 \AA$ in steps of $0.025 \AA$. By elongation of all $\mathrm{C}-\mathrm{C}$ bonds in one benzene monomer and simultaneous compression of all $\mathrm{C}-\mathrm{C}$ bonds in the other one, i.e., following the anti-symmetric distortion, the symmetry of the benzene dimer is reduced from $D_{6 h}$ to $C_{6 v}$. Therefore, calculations were performed in $D_{6 h}$ $\left(r_{\mathrm{CC} 1}=r_{\mathrm{CC} 2}\right)$ and $C_{6 v}\left(r_{\mathrm{CC} 1} \neq r_{\mathrm{CC} 2}\right)$ point groups.

Cuts through the potential energy surfaces obtained at TDDFT/B3LYP-D3 level along the symmetric and anti- 

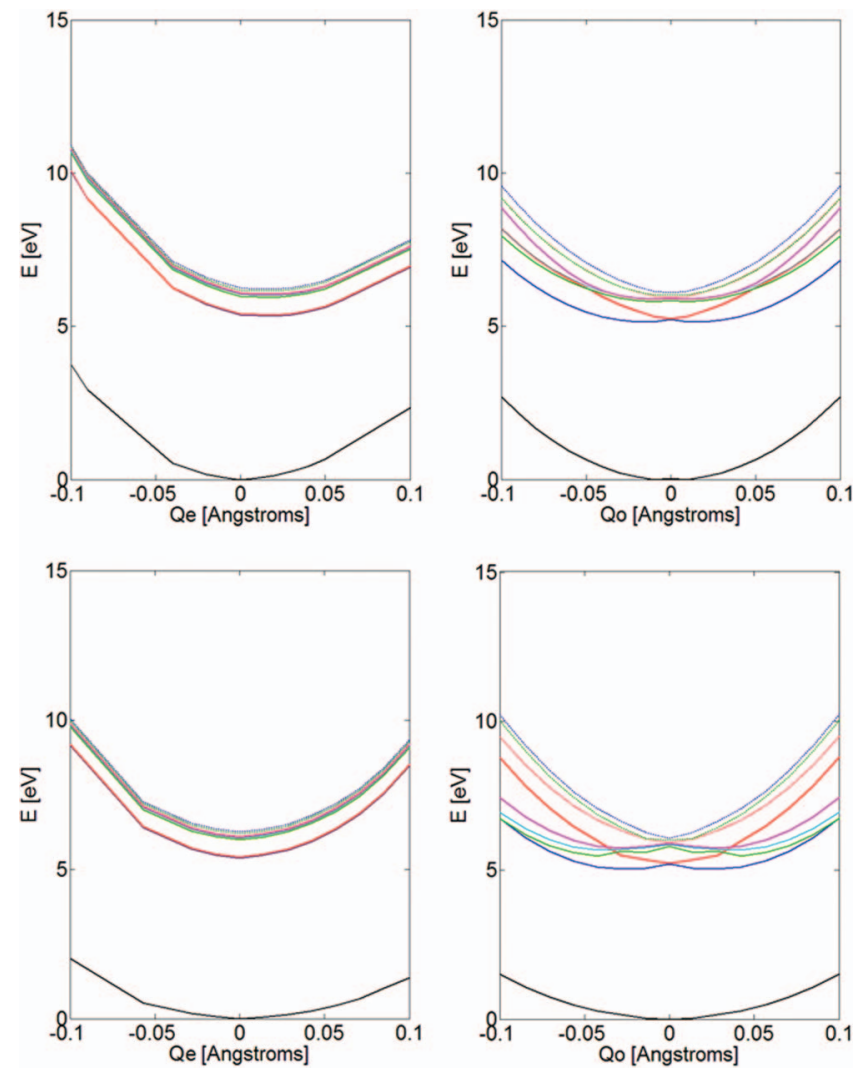

FIG. 10. Potential energy curves of the lowest excited states along the symmetric and the anti-symmetric distortion coordinates $Q_{e}$ (left) and $Q_{o}$ (right) at an intermolecular separation $R$ of $5 \AA$ of the $D_{6 h}$ benzene dimer; TDDFT/B3LYP-D3 calculated plots are given on the top, while the plots obtained using the model Hamiltonian are given at the bottom.

symmetric distortions, at intermolecular separation of 3, 3.89, and $5 \AA$ are displayed in Figures 8-10 (top), respectively. In the same figures (bottom), results obtained using the model Hamiltonian (Eq. (19)) are shown. Remember that all necessary parameters for the model Hamiltonian, except the $\Delta$, are extracted from $a b$ initio calculations of the monomer alone. ${ }^{73}$ However, at large intermolecular separation it is straightforward to derive also $\Delta$ from monomer calculations alone knowing the relative orientation of the monomers using the classical Förster equation for the coupling of the transition dipole moments. ${ }^{74}$ For shorter distances further contributions need to be taken into account. ${ }^{68}$

The overall agreement between $a b$ initio and model curves is excellent, despite the simplicity of the proposed model. The only discrepancy can be found for the slope of the four highest considered excited states in the model, Figures 8-10 (bottom right). This is not surprising, since in pseudo JT theory higher excited states are generally needed to soften these curves. ${ }^{75,76}$ However, for the localization of the exciton, i.e., the correct description of the shape of the ${ }^{I} B_{2 g}$ curve, which is reproduced perfectly, only eight states are needed, which are included in the model. In addition, a slight anharmonicity of the TDDFT computed curves can generally be noted (Figures 8-10, top left). Obviously, this remains undetected in the purely harmonic model (Figures 8-10, bottom left).
At an intermolecular separation of $3 \AA$, the splitting of the first two excited states, ${ }^{1} B_{2 g}$ and ${ }^{1} B_{1 u}$, is apparent (Figure 8 , left). Along the anti-symmetric distortion of the first excited state, only one minimum exists (Figure 8, right). Having a closer look at Figure 8 (top right), the minimum of the curve of the first excited state lies at $r_{\mathrm{CC} 1}=r_{\mathrm{CC} 2}$, though at longer bond lengths of $1.41 \AA$, which is between the equilibrium bond lengths of the benzene molecule in the ground and first excited state.

Moving to the intermolecular separation of $3.89 \AA$, the splitting of the states decreases, as expected (Figure 9, left), because excitonic coupling decreases with intermolecular distance as $R^{-6}$. Regarding the anti-symmetric distortion (Figure 9, top right), the interaction between the two first excited states at the point $r_{\mathrm{CC} 1}=r_{\mathrm{CC} 2}=1.41 \AA$ can be noticed. Consequently, two very weakly visible minima in the first excited state, where one monomer unit has $\mathrm{C}-\mathrm{C}$ bond lengths $1.43 \AA$ and the other $1.39 \AA$ and vice versa, arise, which represent characteristic examples of pseudo JT distortion. This situation can be seen as an intermediate case where weak localization of the exciton due to the pseudo JT distortion can occur. The curve of the first excited state along the antisymmetric distortion coordinate $Q_{o}$ obtained from the model Hamiltonian (Figure 9, bottom right) is in excellent agreement with the ab initio curve.

Finally, at $R=5 \AA$, the first two excited states, ${ }^{1} B_{2 g}$ and ${ }^{1} B_{1 u}$, are practically degenerate (Figure 10 , left). At this intermolecular separation, only a very weak interaction exists between the two monomer units. Removal of the degeneracy is achieved along anti-symmetric distortion and a symmetric double minimum emerges (Figure 10, right). The obtained minima correspond to structures in which one benzene monomer has $\mathrm{C}-\mathrm{C}$ bond lengths of $1.43 \AA$ and the other of $1.39 \AA$ and vice versa. It is obvious that one monomer takes on the equilibrium bond distance of the electronic ground state, while the other the one of the first excited states. In other words, localization of the exciton on one of the monomers in the excited benzene dimer can occur at this intermolecular separation of $5 \AA$.

It should be noted that in this case degeneracy exists by construction, i.e., by putting two equivalent molecules in spatial proximity exhibiting $D_{6 h}$ symmetry. However, the distortion that the system undergoes is not due to the JT effect. The reasons can be found in point group theory as the final state representation must come from the sum of the two initial irreducible representations. Therefore, the subspace of the degenerate $B_{2 g}$ and $B_{1 u}$ states corresponds to a double irreducible representation $\left(B_{2 g}+B_{1 u}\right)$. Taking the direct product with itself, the result is the total symmetric representation $A_{I g}$ plus the asymmetric $A_{2 u}$, which is exactly the connecting mode between the $D_{6 h}$ and $C_{6 v}$ symmetric structures, representing the anti-symmetric distortion mode of the benzene $\mathrm{C}-\mathrm{C}$ bonds. Moreover, the strong mixing of these two initial wave functions strongly changes the initial character of the state transferring charge from one molecule to the other clearly indicating that this is a strong pseudo JTE with a very small gap.

Similar to previous results for the CO dimer, ${ }^{20}$ distance dependent EC can either quench the pseudo JT effect $(R$ $=3 \AA)$, or lead to the formation of pseudo JT $(R=3.89 \AA)$ 
that becomes very strong with quasi-degeneracy at $(R=5 \AA)$. In the two later cases, breaking the symmetry results in asymmetrically distorted molecular geometries with lower energy and concomitant localization of the excitation energy on one monomer unit.

It is noteworthy that the developed simplified model leads to the same conclusions as computationally demanding $a b$ initio calculations, bearing the advantage of giving detailed insight in the behavior of excitonically coupled chromophores while being conceptually simple and computationally cheap.

\section{CONCLUSION}

Excitonic coupling is the physical quantity that determines the function and efficiency of many photo-biological processes like light harvesting or light reception, or of technological devices like organic light emitting diodes or organic photovoltaics. In this work, a novel scheme for analyzing exciton localization or equivalently excitation energy transfer in excitonically coupled dimers taking vibronic coupling into account is presented, which can be derived from intrinsic properties of the constituting monomers alone. The derived model has here been exemplified using the benzene dimer and its validity and applicability have been tested against $a b$ initio results. Most importantly, the derived Hamiltonian forms the basis for more advanced quantum simulations of excitons in coupled chromophores.

Spatially separated equivalent molecules have degenerate excited states by construction. In principle, two possibilities exist to lift the degeneracy, either by lowering the intermolecular separation between two monomer units to increase EC, or by vibronic coupling where only a small displacement of the atoms removes the degeneracy and lowers the energy of the states, indicating strong vibronic coupling in the excited states, whereas the ground state is not affected. In other words, if two chromophores are close enough that excitonic coupling plays the main role for splitting of the excited states, this situation corresponds to the coherent regime of excitation energy transfer as the exciton remains delocalized over both chromophores. With increasing the intermolecular separation, EC strength decreases, and as soon as the vibronic coupling approaches a similar magnitude than EC, pseudo JT distortion occurs and the exciton tends to localize on one monomer. Going to even larger intermolecular separation, a very strong pseudo JT effect removes the excited states degeneracy.

In order to rationalize the effects that are taking place in the process of exciton localization, we have developed this model based on the vibronic coupling theory, to elucidate the mechanism of exciton localization vs. excitation energy transfer, in strong, weak, and intermediate EC case. The key role of the pseudo JT coupling, especially in intermediate EC situations, has been illuminated. In particular, neglecting the pseudo JT coupling between excited states in the model would always lead to a delocalized picture of the exciton.

Bearing in mind the importance of excitation energy transfer, its consideration from a JT perspective will pave the road to a better understanding of interacting chromophores in a large number of biological systems. The purpose of the new developed model is to represent and predict the properties of coupled identical chromophores based on intrinsic features of the constituting monomer alone, allowing us to study much larger biological or chemical systems, but still to achieve microscopic insights into the details of exciton localization vs. excitation energy transfer in complex systems. In other words, our main conclusion is that most of the influence of vibronic and excitonic couplings on the localization of the exciton can be obtained from calculations of a single monomer. This will allow for studying the properties of isolated molecules by looking for systems with improved excitation transfer properties.

\section{ACKNOWLEDGMENTS}

This work was supported by the Serbian Ministry of Education and Science (Grant No. 172035) and the Spanish Ministerio de Industria e Innovación under project FIS201237549-C05-04. Support by the COST-CMTS Action CM1002 "COnvergent Distributed Environment for Computational Spectroscopy (CODECS)" is also gratefully acknowledged.

${ }^{1}$ J. Cornil, D. Beljonne, J.-P. Calbert, and J.-L. Brédas, Adv. Mater. 13, 1053 (2001).

${ }^{2}$ T. O. Pullerits and V. Sundström, Acc. Chem. Res. 29, 381 (1996).

${ }^{3}$ A. C. Grimsdale and K. Müllen, Angew. Chem., Int. Ed. 44, 5592 (2005).

${ }^{4}$ J. C. Sancho-García and A. J. Pérez-Jiménez, J. Chem. Phys. 129, 024103 (2008).

${ }^{5}$ A. Damjanović, I. Kosztin, U. Kleinekathöfer, and K. Schulten, Phys. Rev. E 65, 031919 (2002)

${ }^{6}$ A. Hadipour, B. de Boer, J. Wildeman, F. Kooistra, J. Hummelen, M. Turbiez, M. Wienk, R. Janssen, and P. Blom, Adv. Funct. Mater. 16, 1897 (2006).

${ }^{7}$ G. Gustafsson, Y. Cao, G. M. Treacy, F. Klavetter, N. Colaneri, and A. J. Heeger, Nature (London) 357, 477 (1992).

${ }^{8}$ C. J. Brabec, A. Cravino, D. Meissner, N. S. Sariciftci, T. Fromherz, M. T. Rispens, L. Sanchez, and J. C. Hummelen, Adv. Funct. Mater. 11, 374 (2001).

${ }^{9}$ K. M. Coakley and M. D. McGehee, Chem. Mater. 16, 4533 (2004).

${ }^{10}$ S. R. Forrest, Chem. Rev. 97, 1793 (1997).

${ }^{11}$ P. Peumans, A. Yakimov, and S. R. Forrest, J. Appl. Phys. 93, 3693 (2003).

${ }^{12}$ J.-L. Brédas, D. Beljonne, V. Coropceanu, and J. Cornil, Chem. Rev. 104, 4971 (2004).

${ }^{13}$ J.-L. Brédas, J. Cornil, and A. J. Heeger, Adv. Mater. 8, 447 (1996).

${ }^{14}$ S. E. Shaheen, G. E. Jabbour, B. Kippelen, N. Peyghambarian, J. D. Anderson, S. R. Marder, N. R. Armstrong, E. Bellmann, and R. H. Grubbs, Appl. Phys. Lett. 74, 3212 (1999).

${ }^{15}$ H. E. Katz, A. J. Lovinger, J. Johnson, C. Kloc, T. Siegrist, W. Li, Y. Y. Lin, and A. Dodabalapur, Nature (London) 404, 478 (2000).

${ }^{16}$ Z. Bao, Adv. Mater. 12, 227 (2000).

${ }^{17}$ S.-B. Rim, R. F. Fink, J. C. Schöneboom, P. Erk, and P. Peumans, Appl. Phys. Lett. 91, 173504 (2007).

${ }^{18}$ A. Damjanovic, H. M. Vaswani, P. Fromme, and G. R. Fleming, J. Phys. Chem. B 106, 10251 (2002).

${ }^{19}$ A. Dreuw, G. R. Fleming, and M. Head-Gordon, J. Phys. Chem. B 107, 6500 (2003).

${ }^{20}$ P. H. P. Harbach and A. Dreuw, Chem. Phys. 377, 78 (2010).

${ }^{21}$ W. Zinth and J. Wachtveitl, Chem. Phys. Chem. 6, 871 (2005).

${ }^{22}$ V. May and O. Kühn, Charge and Energy Transfer Dynamics in Molecular Systems, 3rd ed. (Wiley-VCH, Weinheim, 2011).

${ }^{23}$ P. Ottiger, S. Leutwyler, and H. V. Köppel, J. Chem. Phys. 136, 174308 (2012).

${ }^{24}$ P. Ottiger, S. Leutwyler, and H. Köppel, J. Chem. Phys. 131, 204308 (2009).

${ }^{25}$ S. Polyutov, O. Kühn, and T. Pullerits, Chem. Phys. Chem. 394, 21 (2012).

${ }^{26}$ H.-M. Wu, M. Ratsep, I.-J. Lee, R. J. Cogdell, and G. J. Small, J. Phys. Chem. B 101, 7654 (1997). 
${ }^{27}$ V. I. Novoderezhkin, M. A. Palacios, H. van Amerongen, and R. van Grondelle, J. Phys. Chem. B 108, 10363 (2004).

${ }^{28}$ I. B. Bersuker, The Jahn-Teller Effect (Cambridge University Press, 2006).

${ }^{29}$ T. Förster, "Delocalized excitation and excitation transfer," in Modern Quantum Chemistry, edited by O. Sinanoglu (Academic, New York, 1965), p. 93.

${ }^{30}$ J. B. Birks, Rep. Prog. Phys. 38, 903 (1975).

${ }^{31}$ A. Waldman and S. Ruhman, Chem. Phys. Lett. 215, 470 (1993).

${ }^{32}$ T. Hirata, H. Ikeda, and H. Saigusa, J. Phys. Chem. A 103, 1014 (1999).

${ }^{33}$ H. Saigusa, M. Morohoshi, and S. Tsuchiya, J. Phys. Chem. A 105, 7334 (2001).

${ }^{34}$ K. Law, M. Schauer, and E. R. Bernstein, J. Chem. Phys. 81, 4871 (1984).

${ }^{35}$ N. Nakashima, M. Sumitani, I. Ohmine, and K. Yoshihara, J. Chem. Phys. 72, 2226 (1980).

${ }^{36}$ J. T. Richards and J. K. Thomas, Chem. Phys. Lett. 5, 527 (1970).

${ }^{37}$ R. Bonneau, R. Joussot-Dubien, and R. Bensasson, Chem. Phys. Lett. 3, 353 (1969).

${ }^{38}$ R. Cooper and J. K. Thomas, J. Chem. Phys. 48, 5097 (1968).

${ }^{39}$ J. B. Hopkins, D. E. Powers, and R. E. Smalley, J. Phys. Chem. 85, 3739 (1981).

${ }^{40}$ A. Dreuw and M. Head-Gordon, Chem. Rev. 105, 4009 (2005).

${ }^{41}$ A. Dreuw and M. Head-Gordon, Chem. Phys. Lett. 426, 231 (2006).

${ }^{42}$ C. F. Guerra, J. G. Snijders, G. te Velde, and E. Baerends, J. Theor. Chem. Acc. 99, 391 (1998).

${ }^{43}$ G. te Velde, F. M. Bickelhaupt, S. J. A. van Gisbergen, C. F. Guerra, E. J. Baerends, J. G. Snijders, and T. Ziegler, J. Comput. Chem. 22, 931 (2001).

${ }^{44}$ ADF2010.01, SCM, Theoretical Chemistry, Vrije Universiteit Amsterdam, The Netherlands, 2010, see http://www.scm.com.

${ }^{45}$ A. D. Becke, J. Chem. Phys. 98, 5648 (1993).

${ }^{46}$ P. J. Stephens, F. J. Devlin, C. F. Chabalowski, and M. J. Frisch, J. Phys. Chem. 98, 11623 (1994).

${ }^{47}$ S. Grimme, S. Ehrlich, and L. Goerigk, J. Comput. Chem. 32, 1456 (2011).

${ }^{48}$ E. Runge and E. K. U. Gross, Phys. Rev. Lett. 52, 997 (1984).

${ }^{49}$ M. E. Casida, in Recent Advances in Density Functional Methods, edited by D. P. Chong (World Scientific, Singapore, 1995), p. 155.

${ }^{50}$ A. D. Becke, Phys. Rev. A 38, 3098 (1988).

${ }^{51}$ J. P. Perdew, Phys. Rev. B 33, 8822 (1986).

${ }^{52}$ J. P. Perdew, A. Ruzsinszky, G. I. Csonka, O. A. Vydrov, and G. E. Scuseria, Phys. Rev. Lett. 100, 136406 (2008).

${ }^{53}$ O. V. Gritsenko, P. R. T. Schipper, and E. J. Baerends, Chem. Phys. Lett. 302, 199 (1999).

${ }^{54}$ P. R. T. Schipper, O. V. Gritsenko, S. J. A. van Gisbergen, and E. J. Baerends, J. Chem. Phys. 112, 1344 (2000).

${ }^{55}$ S. Grimme, J. Comput. Chem. 25, 1463 (2004).

${ }^{56}$ M. Ernzerhof and G. Scuseria, J. Chem. Phys. 110, 5029 (1999).

${ }^{57}$ J. Gauss and J. F. Stanton, J. Phys. Chem. A 104, 2865 (2000).

${ }^{58}$ A. Hiraya and K. Shobatake, J. Chem. Phys. 94, 7700 (1991).
${ }^{59}$ N. Nakashima, H. Inoue, M. Sumitani, and K. Yoshihara, J. Chem. Phys. 73, 5976 (1980).

${ }^{60}$ J. P. Doering, J. Chem. Phys. 51, 2866 (1969).

${ }^{61}$ R. Astier and Y. H. Meyer, Chem. Phys. Lett. 3, 399 (1969).

${ }^{62}$ E. N. Lassettre, A. Skerbele, M. A. Dillon, and K. J. Ross, J. Chem. Phys. 48, 5066 (1968).

${ }^{63}$ T. Hashimoto, H. Nakano, and K. Hirao, J. Chem. Phys. 104, 6244 (1996).

${ }^{64}$ T. Hashimoto, H. Nakano, and K. Hirao, J. Mol. Struct.: THEOCHEM 451, 25 (1998)

${ }^{65}$ C. Adamo, G. E. Scuseria, and V. Barone, J. Chem. Phys. 111, 2889 (1999).

${ }^{66}$ M. Schreiber, M. R. Silva-Junior, S. P. A. Sauer, and W. Thiel, J. Chem. Phys. 128, 134110 (2008).

${ }^{67}$ K. Diri and A. I. Krylov, J. Phys. Chem. A 116, 653 (2012).

${ }^{68}$ R. F. Fink, J. Pfister, H. M. Zhao, and B. Engels, Chem. Phys. 346, 275 (2008).

${ }^{69}$ M. Nooijen, Spectrochim. Acta, Part A 55, 539 (1999).

${ }^{70}$ T. C. Dinadayalane and J. Leszczynski, Struct. Chem. 20, 11 (2009).

${ }^{71}$ P. Hobza, V. Spirko, H. L. Selzle, and E. W. Schlag, J. Phys. Chem. A 102, 2501 (1998).

${ }^{72}$ T. Rocha-Rinza, L. De Vico, V. Veryazov, and B. O. Roos, Chem. Phys. Lett. 426, 268-272 (2006).

${ }^{73}$ The obtained force of the dimer at small $R$ corresponds to the force derived from Eq. (12) and can be presented through the force calculated for the monomer. At intermolecular separation of $3 \AA$, the force obtained by TDDFT calculations of the dimer is $5.68 \mathrm{eV} / \AA$, and corresponds to the value of $6.01 \mathrm{eV} / \AA ̊$ obtained using Eq. (12) in the model. With increasing the separation between monomer units total force that drives the nuclei to the minimum increases, and at $R=3.89 \AA$ it has the value of $6.96 \mathrm{eV} / \AA$. The total force at intermolecular distance of $5 \AA$ is $8.54 \mathrm{eV} / \AA$, which is in the excellent agreement with the force obtained for the monomer in the first excited state $(8.48 \mathrm{eV} / \AA$ ) $) . K_{e}$ and $K_{o}$ were obtained from the monomer force constant in the excited state. Following the model and Eq. (15) the dimer force constants are equal to the monomer force constant. $K_{\text {mono }}$ has a value of $313.07 \mathrm{eV} / \AA^{2}$. The dimer forces, $K_{e}$ and $K_{o}$, where the monomer units are strongly separated, are $318.10 \mathrm{eV} / \AA^{2}$ and $269.82 \mathrm{eV} / \AA^{2}$, respectively. Moving to smaller intermolecular separations, $K_{e}$ and $K_{o}$ have almost constant values. At intermolecular distance of $3.89 \AA \mathrm{Ke}$ is $316.49 \mathrm{eV} / \AA^{2}$ and at $R=3 \AA$ it has value of $318.28 \mathrm{eV} / \AA^{2} . K_{o}$ calculated at intermolecular separation of $3.89 \AA$ is $262.43 \mathrm{eV} / \AA^{2}$ and at intermolecular separation of $3 \AA$ the constant has a value of $283.45 \mathrm{eV} / \AA^{2}$.

${ }^{74}$ T. Förster, Ann. Phys. 437, 55 (1948).

${ }^{75}$ P. García-Fernández and I. B. Bersuker, Phys. Rev. Lett. 106, 246406 (2011).

${ }^{76}$ P. García-Fernández, Y. Liu, I. B. Bersuker, and J. E. Boggs, Phys. Chem. Chem. Phys. 13, 3502 (2011). 